\title{
IBD in the time of corona - vigilance for immune-mediated diseases
}

Alexander R. Moschen (i)

The gastrointestinal tract represents a target organ for severe acute respiratory syndrome coronavirus 2 (SARS-CoV-2), warranting a thorough discussion on the consequences, particularly regarding inflammatory bowel diseases. Here, aspects of gastrointestinal involvement with SARS-CoV-2, the role of viruses as modulators of mucosal immunity and as treatment-related safety hazards, and the current clinical evidence will be discussed.

In the past few months, the coronavirus disease 2019 (COVID-19) pandemic caused by novel severe acute respiratory syndrome coronavirus 2 (SARS-CoV-2) infection has had the world on tenterhooks. From a number of perspectives, this unprecedented situation has taught us lessons economically, socially and clinically. Even though infection with SARS-CoV-2 induces mild flu-like symptoms in the majority of those affected, some patients will develop severe COVID-19, with progressive pneumonia, acute respiratory distress syndrome and organ failure due to an overreacting immune system. Unsurprisingly, this situation also poses major challenges for gastroenterologists, particularly for the management of inflammatory bowel diseases (IBD) (BOX 1). Caring for our patients' safety in the current situation from the start raised important questions: whether COVID-19 might worsen pre-existing IBD or even trigger IBD, whether patients with IBD belong to a group at risk of a complicated course of the disease and, particularly, the potential threat emanating from immunosuppressive and biologic agents that we use to treat IBD.

SARS-CoV-2 spreads primarily through droplets of saliva or nasal discharges, making the upper respiratory tract the major site of entry; concerns remain about viral spread from asymptomatic individuals in addition to infected individuals before developing symptoms. On the basis of sequence homologies and knowledge gained from the existing SARS-CoV, it has been shown that SARS-CoV-2 gains access to target cells through the interaction of the virus surface spike (S) protein with the host membrane-bound angiotensin I converting enzyme 2 (ACE2). Cleavage of the $S$ protein by host proteases, including TMPRSS2, TMPRSS4 and CatB/L, facilitates viral uptake ${ }^{1}$. Both ACE2 and the TMPRSS2 proteases are currently being targeted in clinical studies. As well as being highly expressed in the lung (lower airways), ACE2 is expressed on the luminal surface of intestinal epithelial cells (IECs) lining the gut mucosa. Unsurprisingly, patients with COVID-19 can develop gastrointestinal symptoms including nausea and vomiting and diarrhoea ${ }^{1}$. Diarrhoea seems to be directly related to the infection of IECs, as Lamers and colleagues demonstrated that SARS-CoV-2 is indeed capable of infecting and replicating in differentiated human enterocytes of small intestinal organoids, where it induces a strong antiviral transcriptional programme ${ }^{2}$. Notably, RNA from SARS-CoV-2 was detected in the faeces of patients with COVID-19, raising the possibility of transmission through a faecal-oral route ${ }^{1}$.

So what are the potential consequences of such evidence on mucosal immunity in IBD? Viruses are abundant pathogens and part of our intestinal microbiota. Experimental evidence suggests that some eukaryotic viruses can spark or modulate intestinal inflammation in a genetically susceptible host. Mice carrying a hypomorphic Atg16l1 gene develop Paneth cell abnormalities only in the presence of persistent murine norovirus, which alters mucosal immune responses following subsequent injuries ${ }^{3}$. Reoviruses have been shown to interfere with tolerogenic immune responses to dietary antigens, thus promoting the onset and perpetuation of coeliac disease $^{4}$. The potential of SARS-CoV-2 to trigger chronic intestinal immunity remains to be determined; however, reports of a hyperinflammatory Kawasaki-like disease in children highlight that immune-mediated disease in association with COVID-19 is conceivable ${ }^{5}$.

Could SARS-CoV-2 affect intestinal immunity or beyond in pre-existing IBD? Clinical experience has shown that severe secondary bacterial infections can occur after viral disease. The mechanisms underlying virus-induced immunosuppression remain a subject of research. After entering the host target cell, SARS-CoV-2 is released into the cytoplasm to enable replication of the viral genome and the assembly and release of new virions. Here, the viral RNA is recognized by members of the RIG-like receptor family, including RIG-I and IFIH1 (MDA5), activating transcriptional regulators of the type I interferon pathway. Notably, IFIH1 has been identified as a risk gene for $\mathrm{IBD}^{6}$. Besides members of 


\section{Box $1 \mid$ Key messages and perspectives}

- Current observations do not suggest an increased risk of infection with severe acute respiratory syndrome coronavirus 2 (SARS-CoV-2) or an unfavourable course of coronavirus disease 2019 (COVID-19) in patients with inflammatory bowel disease (IBD).

- Based on the currently available data, immunomodulatory and biologic agents used for the treatment of IBD do not seem to be associated with an increased risk of severe COVID-19 or COVID-19-associated mortality.

- As studies suggest that disease activity and the use of corticosteroids, arguably reflecting disease activity, might increase the mortality, patients with IBD should receive medical treatment adhering to current clinical guidelines.

- To substantiate available clinical evidence in this highly dynamic situation, any new cases of COVID-19 among patients with IBD should be continued to be consequently registered (https://covidibd.org/).

- As the currently available data are mainly based on case collections, a reporting bias is likely. Future epidemiological research using serological testing in larger cohorts of patients with IBD and other immune-mediated diseases along with matched controls will help to further dissect the risk associated with the underlying disease or respective therapies.

- Comparative immunological studies using organoid cultures from patients with IBD and healthy individuals as controls could help to decipher whether a genetic underpinning associated with IBD might affect innate immune responses towards SARS-CoV-2, therefore helping to identify general genetic risk driving severe COVID-19.

- The availability of an effective vaccine against SARS-CoV-2 is eagerly awaited. The developmental activities should ideally include patients on immunosuppression early on to ensure protection for those who need it most urgently.

the IL-1 family, type I interferons are part of the first line of defence and an important link between innate and adaptive immunity. Studies have shown that type I interferons potently suppress $T$ helper $17\left(\mathrm{~T}_{\mathrm{H}} 17\right)$ cell differentiation, thereby interfering with critical antibacterial and antifungal cytokines such as IL-17 (REF.). It is currently unclear whether defects or even an overactivation of the early type I interferon immune response contributes to the transition from non-severe to severe COVID-19, although trials are underway of antiviral therapy that includes IFN $\beta$ (NCT04276688, NCT04354259).

In the management of IBD, viral infections are part of the daily clinical routine. Unsurprisingly, viruses, particularly members of the herpes virus group, including herpes simplex, herpes zoster and cytomegalovirus, are among the most common pathogens causing opportunistic infections. Such a risk increases with the extent of immunosuppression and is greatest in patients on triple combinations, particularly when corticosteroids are in use ${ }^{8}$. On the other hand, we have learned about drug class-specific complications, meaning that specific immunosuppressants can drive the risk of specific pathogens. For instance, tumour necrosis factor (TNF) antagonists increase the risk of hepatitis B virus reactivation, and natalizumab (targeting $\alpha 4$ integrin) has been shown to cause progressive multifocal leukoencephalopathy by reactivation of the John Cunningham virus $^{8}$. Taking this knowledge into consideration, the heightened concerns from the beginning seem comprehensible and prudent. A relevant case series of 79 patients, $>50 \%$ receiving biologic agents including TNF blockers, vedolizumab (anti- $\alpha 4 \beta 7$ ) and ustekinumab (anti-IL12-anti-IL-23) and 11\% under systemic corticosteroids, has been reported from Italy suggesting that COVID-19 mortality among patients with IBD is associated with older age, comorbidities and active disease but not with IBD treatment ${ }^{9}$. Additional information is coming from an international COVID-19 IBD registry (SECURE-IBD database) that has been supported by the International Organization for the Study of IBD. To date, as of 9 June 2020, 1,439 cases have been registered. The results from the first 525 cases are now published $^{10}$, and the information provided is largely confirmative of the Italian observations. These reassuring safety data might prompt efforts to utilize immunosuppressive drugs, targeting TNF, IL-1, IL-12-IL-23 or IL-6, in patients who develop severe respiratory and systemic COVID-19 that is driven and maintained by a cytokine release syndrome. In light of the considerable mortality of severe COVID-19 and recalling older well-conducted trials addressing cytokines such as TNF in sepsis, it is advisable to test such approaches in properly performed and controlled trials.

In general, it is by examining past experience that makes it possible to adjudge the sense or nonsense of challenging clinical decisions. One of the few positive aspects of this pandemic seems to me to be that we are managing to unite common objectives to pool interests, to generate smart ideas, and to promote international collaboration. Thus, within a few weeks, joint efforts by the IBD community worldwide have laid the foundations on the basis of which we are already able to make better and safer clinical decisions for our patients with IBD and hopefully to help in the fight against this new coronavirus.

1. Neurath, M. F. Covid-19 and immunomodulation in IBD. Gut https://doi.org/10.1136/gutjnl-2020-321269 (2020).

2. Lamers, M. M. et al. SARS-CoV-2 productively infects human gut enterocytes. Science https://doi.org/10.1126/science.abc1669 (2020).

3. Cadwell, K. et al. Virus-plus-susceptibility gene interaction determines Crohn's disease gene Atg 16L1 phenotypes in intestine. Cell 141, 1135-1145 (2010).

4. Bouziat, R. et al. Reovirus infection triggers inflammatory responses to dietary antigens and development of celiac disease. Science 356, 44-50 (2017).

5. Verdoni, L. et al. An outbreak of severe Kawasaki-like disease at the Italian epicentre of the SARS-CoV-2 epidemic: an observational cohort study. Lancet https://doi.org/10.1016/S0140-6736(20)31103-X (2020).

6. Jostins, L. et al. Host-microbe interactions have shaped the genetic architecture of inflammatory bowel disease. Nature 491, 119-124 (2012).

7. Tilg, H., Moschen, A. R. \& Kaser, A. Suppression of interleukin-17 by type I interferons: a contributing factor in virus-induced immunosuppression? Eur. Cytokine Netw. 20, 1-6 (2009)

8. Ali, T., Yun, L., Shapiro, D., Madhoun, M. F. \& Bronze, M. Viral infections in patients with inflammatory bowel disease on immunosuppressants. Am. J. Med. Sci. 343, 227-232 (2012)

9. Bezzio, C. et al. Outcomes of COVID-19 in 79 patients with IBD in Italy: an IG-IBD study. Gut https://doi.org/10.1136/gutjnl-2020321411 (2020).

10. Brenner, E. J. et al. Corticosteroids, but not TNF antagonists, are associated with adverse COVID-19 outcomes in patients with inflammatory bowel diseases: results from an international registry. Gastroenterology https://doi.org/10.1053/j.gastro.2020.05.032 (in press).

\section{Competing interests}

The author declares no competing interests.

\section{RELATED LINKS}

ClinicalTrials.gov: https://clinicaltrials.gov/

SECURE-IBD database: https://covidibd.org/ 